\title{
Correction to: A priori bounds for the kinetic DNLS
}

Nobu Kishimoto and Yoshio Tsutsumi

\section{Correction to:}

Chapter "A priori bounds for the kinetic DNLS" in:

D. R. Wood et al. (eds.), 2019-20 MATRIX Annals, MATRIX Book Series 4, https://doi.org/10.1007/978-3-030-62497-2_63

The original version of this chapter was revised in online version, the following correction has been incorporated: The author name "Federico Cacciafesta" has been changed to "Nobu Kishimoto" in Chapter 63. The chapter has been updated with the changes. 